\title{
Locally Advanced Osteosarcoma of the Ethmoid Sinus: A Report of Successful Management
}

\author{
Swathi Gopishetty ${ }^{a}$ Justin Yeh ${ }^{b}$ Mansoor Khalid ${ }^{c}$ Pavan Annamaraju $^{d}$ \\ Achuta K. Guddati ${ }^{\mathrm{e}}$

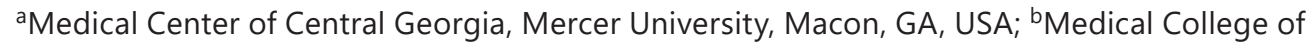 \\ Georgia, Augusta University, Augusta, GA, USA; 'Mercy Hospital Oklahoma City, Oklahoma \\ City, OK, USA; dLoma Linda Medical Center, Loma Linda University School of Medicine, \\ Loma Linda, CA, USA; 'Division of Hematology/Oncology, Georgia Cancer Center, Augusta \\ University, Augusta, GA, USA
}

Keywords

Adjuvant chemotherapy · Cancer · Metastasis · Osteosarcoma · Otorhinolaryngology

\section{Abstract}

Osteosarcoma of the skull has poor outcomes. This case report describes the presentation and clinical course of a patient who was diagnosed with osteosarcoma of the skull involving the cribriform plate. After her initial diagnosis, she developed esotropia with severe unremitting headaches. She received palliative radiation, followed by chemotherapy, and responded well. Her initial symptoms involving the cranial nerves subsided, and her response was sustained. This report illustrates the need to effectively treat osteosarcoma of the skull despite its reported poor outcomes.

\section{Introduction}

Osteosarcoma is a malignant tumor with a rare occurrence in the sinonasal region. Osteosarcomas of the head and neck region commonly occur in the mandible and maxilla, but occurrence in the ethmoid sinus is a very rare entity. Primary osteosarcoma in the nasal cavity and paranasal sinus account for about $0.5-8.1 \%$ of osteosarcoma [1]. Due to the rarity of the disease occurrence, rigorous studies are challenging to conduct to determine the treatment plan. Herein, we present a case of an aggressive sinonasal high-grade osteosarcoma of the osteoblastic subtype. 
Gopishetty et al.: Osteosarcoma of the Cribriform Plate

\section{Case Presentation}

A 55-year-old African American woman initially presented with a nose bleed and a syncopal episode, which was adequately managed with nasal packing. She also complained of sinus congestion for several weeks, and rigid nasal endoscopy by the otorhinolaryngology service showed edema on the left nasal cavity and hematoma of the inferior turbinate. During a follow-up visit, it was noted that even though her epistaxis had improved, she continued to have nonbloody nasal discharge. During further follow-up clinic visits, she complained of left facial fullness, eye pain, blurred vision, and left strabismus with diplopia. Computed tomography $(\mathrm{CT})$ scan was done, and she was found to have a large $(6.7 \times 3.5 \times 3.0 \mathrm{~cm})$ lobulated partially calcified soft tissue mass at the nasal cavity/anterior skull base. It was centered in the skull base extending through the ethmoid and left nasal cavity anteriorly and clivus posteriorly with likely intracranial extension through the cribriform plate, the sellar and prepontine cistern regions mostly involving the nasal canal and likely the left foramen rotundum. Magnetic resonance imaging (MRI) showed a heterogeneously enhancing mass centered within the left nasal cavity, left ethmoid, cribriform plate, left cavernous sinus and clivus abutting the posterior medial orbital apices with the extra-axial dural extension and associated perineural spread involving the left V2 and possibly medial aspect of left V3. Flexible endoscopy identified a left nasal cavity mass that was biopsied. The pathology report confirmed the diagnosis of osteosarcoma, high-grade osteoblastic type. A positron emission tomography (PET) scan demonstrated no evidence of distant metastasis. The tumor was staged as cT4bNOM0.

Her physical examination was pertinent for left diplopia and blurred vision, which was associated with cranial nerve VI, left 3rd nerve causing partial ptosis, left V2, and left 6th nerve palsy. Her symptoms initially resolved with dexamethasone administration. Soon after that, she developed facial swelling, dyspnea, dysphagia, worsening diplopia, and headaches. After her case was discussed in the multidisciplinary tumor board, she was started on cisplatin and doxorubicin as induction chemotherapy. Percutaneous gastrostomy tube was placed due to dysphagia. She appeared to have responded initially; however, during a follow-up visit, her CT confirmed disease progression. MRI also showed an interval increase in tumor size (previously $5.8 \times 3.6 \times 3.8 \mathrm{~cm}$ and now $8.4 \times 3.9 \times 4.2 \mathrm{~cm}$ ). The tumor was then staged as T4N1M0. Due to worsening symptoms, she received 5 fractions of palliative radiation therapy following which a slight reduction in the tumor size was observed. She then received a 2 nd cycle of chemotherapy and continued to remain on dexamethasone. Pegfilgrastim was also initiated for supportive therapy.

Subsequently, our patient had to move out of our service area due to family reasons, prompting transfer of medical care to another local institution. Meanwhile, her chemotherapy regimen was changed to gemcitabine and docetaxel. She was seen back at our facility for the 3rd cycle of gemcitabine and docetaxel. She was noted to have a significant improvement in her symptoms. Further, the repeat MRI demonstrated stable disease. Other than mild fatigue, she continued to do well. Her dysphagia resolved, and the percutaneous gastrostomy tube was removed.

\section{Discussion}

Osteosarcoma in the head and neck region is a very aggressive tumor and constitutes a fraction of all the osteosarcomas [2]. It consists of mesenchymal cells that produce osteoid or immature bone. The survival rate is low when compared to other types of head and neck tumors like squamous cell carcinoma [3]. The usual age of occurrence of the primary tumors 
is about in the thirties to forties, which is later than the osteosarcomas of the long bones [4]. The presentation and clinical course of osteosarcoma of the jaw (the most common head and neck site) and non-jaw locations are distinct [5].

Osteosarcoma most commonly occurs as a secondary tumor, which could be due to radiation therapy, chemotherapy, benign bone diseases like Paget's disease, and fibrous dysplasia. Most studies showed male predominance for the disease occurrence [6].

The most common histologic variant is an osteoblastic variant (35-50\%) followed by chondroblastic variant and low-grade osteosarcoma [7]. Osteosarcomas are divided into further subtypes. According to the degree of differentiation, they are categorized as highgrade, telangiectatic osteosarcoma, and low-grade osteosarcoma. Hematogenous metastasis is less frequent in high-grade sinonasal osteosarcoma when compared to osteosarcoma of the long bones. The main concern for this type of malignancy is the involvement of neighboring structures, which include orbit, nasopharynx, cranium, and cranial nerve involvement [8]. The clinical presentation depends on the location and the extent of tumor burden. The involvement of the laryngeal primaries can cause hoarseness of voice. Oropharyngeal tumors can cause dysphagia. While a painless mass is most common, epistaxis, nasal obstruction, or cranial nerve deficits can be present with skull base tumors [9]. Evaluation involves a complete head and neck examination with a focus on cranial nerve assessment to determine any involvement. All radiologic studies are crucial for the detection of primary and metastatic lesions. MRI is superior to CT for characterizing the lesions. MRI is used for local staging, helps delineate soft tissue involvement and intraosseous extension. The workup for tumor staging includes a whole-body bone scan, chest radiograph, and a CT scan of the chest.

The mainstay of treatment is wide excision with a strong consideration for postsurgical radiation. As head and neck sarcomas have a poor prognosis and high postoperative recurrence, radiation therapy can possibly improve overall survival [10]. Adjuvant systemic chemotherapy is given after postoperative radiation and is recommended for tumors that are at high risk for distant metastases. The standard chemotherapy regimen used is methotrexate, adriamycin, and cisplatin (MAP) $[11,12]$. Neoadjuvant chemotherapy is not recommended in low-grade osteosarcoma on the head and neck but appears to be beneficial for the high-grade variety [13].

Due to the anatomical features of the head and neck, achieving negative margins from surgical resection is difficult. Often, surgical margins can be microscopically positive. Negative margins serve as prognostic factors [14]. Local recurrence is more frequent than distant metastases compared to extremity osteosarcoma $[8,15]$.

Radiation therapy plays a significant role in decreasing the disease burden when surgery is not feasible. It can be provided as an adjunctive modality and can be administered either before or after the surgery [16]. Due to the rarity of the disease and the presence of many histologic subtypes, there are conflicting results in multiple case series. Further absence of large phase III clinical trials makes it a difficult disease to treat, especially in the recurrent setting.

This case illustrates the possibility of a favorable outcome in the treatment of skull osteosarcoma with radiation and chemotherapy even when the tumor is unresectable due to its anatomy and is symptomatic due to the central nervous system invasion.

\section{Statement of Ethics}

The patient has consented to the publication of their clinical history and case details. 
Gopishetty et al:: Osteosarcoma of the Cribriform Plate

\section{Disclosure Statement}

No conflicts of interest exist for all the authors.

\section{Funding Sources}

The authors declare that there was no funding for this study.

\section{Author Contributions}

Swathi Gopishetty: literature review; wrote the manuscript, revised manuscript. Justin Yeh: literature review; wrote the manuscript, revised manuscript. Mansoor Khalid: literature review; revised manuscript. Pavan Annamaraju: literature review; wrote the manuscript, revised manuscript. Achuta K. Guddati: literature review; wrote the manuscript, revised manuscript. All authors have read the manuscript and agree to the content.

\section{References}

1 Gonzalez ME, Raghavan P, Cho B, Muttikkal TJ, Rehm PK. Primary osteogenic osteosarcoma of the ethmoid sinus in an adolescent: case report. J Radiol Case Rep. 2016;10(2):1-9.

2 Gadwal SR, Gannon FH, Fanburg-Smith JC, Becoskie EM, Thompson LD. Primary osteosarcoma of the head and neck in pediatric patients: a clinicopathologic study of 22 cases with a review of the literature. Cancer. 2001; 91(3):598-605.

3 Smith RB, Apostolakis LW, Karnell LH, Koch BB, Robinson RA, Zhen W, et al. National Cancer Data Base report on osteosarcoma of the head and neck. Cancer. 2003;98(8):1670-80.

4 Ha PK, Eisele DW, Frassica FJ, Zahurak ML, McCarthy EF. Osteosarcoma of the head and neck: a review of the Johns Hopkins experience. Laryngoscope. 1999;109(6):964-9.

5 van den Berg H, Schreuder WH, de Lange J. Osteosarcoma: a comparison of jaw versus nonjaw localizations and review of the literature. Sarcoma. 2013;2013:316123.

6 Mirabello L, Troisi RJ, Savage SA. International osteosarcoma incidence patterns in children and adolescents, middle ages and elderly persons. Int J Cancer. 2009;125(1):229-34.

7 Klein MJ, Siegal GP. Osteosarcoma: anatomic and histologic variants. Am J Clin Pathol. 2006;125(4):555-81.

8 Misaghi A, Goldin A, Awad M, Kulidjian AA. Osteosarcoma: a comprehensive review. SICOT J. 2018;4:12.

9 Gil Z, Patel SG, Singh B, Cantu G, Fliss DM, Kowalski LP, et al; International Collaborative Study Group. Analysis of prognostic factors in 146 patients with anterior skull base sarcoma: an international collaborative study. Cancer. 2007;110(5):1033-41.

10 Han S, Yin X, Xu W, Wang Y, Han W. The management of head and neck sarcoma. J Craniofac Surg. 2020;31(2): e189-e192.

11 Ferrari S, Palmerini E. Chemotherapy of osteosarcoma. In: Picci P, Manfrini M, Donati DM, Gambarotti M, Righi A, Vanel D, et al. Diagnosis of musculoskeletal tumors and tumor-like conditions: clinical, radiological and histological correlations -- The Rizzoli Case Archive. Cham: Springer International Publishing; 2020. p. 217-21.

12 Yu D, Zhang S, Feng A, Xu D, Zhu Q, Mao Y, et al. Methotrexate, doxorubicin, and cisplatinum regimen is still the preferred option for osteosarcoma chemotherapy: a meta-analysis and clinical observation. Medicine (Baltimore). 2019;98(19):e15582.

13 Boon E, van der Graaf WT, Gelderblom H, Tesselaar ME, van Es RJ, Oosting SF, et al. Impact of chemotherapy on the outcome of osteosarcoma of the head and neck in adults. Head Neck. 2017;39(1):140-6.

14 Granados-Garcia M, Luna-Ortiz K, Castillo-Oliva HA, Villavicencio-Valencia V, Herrera-Gómez A, MosquedaTaylor A, et al. Free osseous and soft tissue surgical margins as prognostic factors in mandibular osteosarcoma. Oral Oncol. 2006;42(2):172-6.

15 Lindford A, McIntyre B, Marsh R, MacKinnon CA, Davis C, Tan ST. Outcomes of the treatment of head and neck sarcomas in a tertiary referral center. Front Surg. 2015;2:19.

16 Schwarz R, Bruland O, Cassoni A, Schomberg P, Bielack S. The role of radiotherapy in osteosarcoma. Cancer Treat Res. 2009;152:147-64. 